\title{
A Systematic Review and Meta-Analysis of Interventions to Prevent Hepatitis C Virus Infection in People Who Inject Drugs
}

\author{
Holly Hagan, ${ }^{1}$ Enrique R. Pouget, ${ }^{2}$ and Don C. Des Jarlais ${ }^{3}$ \\ ${ }^{1}$ New York University College of Nursing, ${ }^{2}$ National Development and Research Institutes, and ${ }^{3}$ Baron Edmond de Rothschild Chemical Dependency \\ Institute, Beth Israel Medical Center, New York, New York
}

Introduction. High rates of hepatitis C virus (HCV) transmission are found in samples of people who inject drugs (PWID) throughout the world. The objective of this paper was to meta-analyze the effects of risk-reduction interventions on HCV seroconversion and identify the most effective intervention types.

Methods. We performed a systematic review and meta-analysis of published and unpublished studies. Eligible studies reported on the association between participation in interventions intended to reduce unsafe drug injection and HCV seroconversion in samples of PWID.

Results. The meta-analysis included 26 eligible studies of behavioral interventions, substance-use treatment, syringe access, syringe disinfection, and multicomponent interventions. Interventions using multiple combined strategies reduced risk of seroconversion by $75 \%$ (pooled relative risk, .25; 95\% confidence interval, .07-.83). Effects of single-method interventions ranged from .6 to 1.6.

Conclusions. Interventions using strategies that combined substance-use treatment and support for safe injection were most effective at reducing HCV seroconversion. Determining the effective dose and combination of interventions for specific subgroups of PWID is a research priority. However, our meta-analysis shows that HCV infection can be prevented in PWID.

Preventing hepatitis C virus (HCV) infection in people who inject drugs (PWID) is a tremendous public health challenge. HCV is highly efficiently transmitted via parenteral exposure to infectious blood, and the prevalence of $\mathrm{HCV}$ infection in PWID typically ranges between $40 \%$ and $90 \%$, depending on geographic location and duration of exposure to injection drug use $[1,2]$. Extremely high HCV prevalence rates-between $85 \%$ and 98\%-have been reported [3-5]. Most reports of HCV incidence in PWID fall into the range of 20-40 infections per 100 PY [6-10]. However, incidence rates above and

Received 22 October 2010; accepted 10 February 2011

Potential conflicts of interest: none reported.

Correspondence: Holly Hagan, PhD, Senior Research Scientist, College of Nursing, New York University, 726 Broadway, 10th floor, New York, NY 10003 (hh50@nyu.edu).

The Journal of Infectious Diseases 2011;204:74-83

(C) The Author 2011. Published by Oxford University Press on behalf of the Infectious Diseases Society of America. All rights reserved. For Permissions, please e-mail: journals.permissions@oup.com

0022-1899 (print)/1537-6613 (online)/2011/2041-0012\$14.00

DOI: 10.1093/infdis/jir196 below this range have been recorded, with some of the highest rates observed in recent-onset drug injectors and in low- and middle-income countries [2, 11-14].

Acute HCV infection results in chronic carriage in $70 \%-80 \%$ of cases, and $20 \%-25 \%$ of those with persistent infection will develop liver disease that may manifest as cirrhosis, liver failure, or hepatocellular carcinoma [15]. In the United States, it is expected that HCV-related mortality will surpass HIV-related mortality in the coming years [16]. No vaccine exists to prevent HCV infection [17]. Treatment for HCV infection is costly, and PWID are less likely to receive medical monitoring and treatment of the infection than other patient groups $[17,18]$. Thus, the prevention of primary HCV infection among PWID is a public health issue of major importance, and behavioral and structural interventions are needed to prevent HCV transmission among PWID. This paper describes the systematic review and meta-analysis of the association between HCV seroconversion and interventions that are intended to reduce injection-related acquisition of $\mathrm{HCV}$ among individuals. 


\section{METHODS}

We collected the data for these analyses as part of the HCV Synthesis Project, a systematic review and meta-analysis of HCV epidemiology and prevention in drug users [19]. The scope of the HCV Synthesis Project includes published and unpublished reports describing the epidemiology of HCV infection in PWID and in non-injection drug users who sniff or smoke heroin, cocaine, or amphetamine. These 2 groups of drug users have been identified as having a biologically plausible risk of exposure to HCV infection via drug-use practices, either through percutaneous or mucous membrane exposure to blood via sharing of straws or through pipes used to administer drugs $[1,20]$. To be eligible for inclusion in the HCV Synthesis Project, studies must have reported HCV prevalence or incidence rates, measures of association with prevalence or incidence, HIV-HCV coinfection rates, or HCV genotype distributions in samples of eligible drug users. In addition, HCV status must have been determined by serologic testing of either sera or oral fluids; oral fluid tests have been shown to have high sensitivity (92\%) and specificity (99\%) [21].

Data collection and abstraction methods have been described in detail elsewhere [19]. We performed automated searches on electronic databases of the published medical literature, the proceedings of scientific conferences, and government and other Web sites related to public health, HIV, hepatitis, and drug use or control. Manual search methods included footnote chasing and searching of related journals and abstract books. We also sought unpublished studies by contacting investigators who were conducting or who had completed studies of HCV epidemiology or prevention. Reports published or released between January 1989 and December 2006 were included in an initial search that identified a total of 2375 reports that we screened for eligibility. Of these, 628 were determined to be eligible and were included in the HCV Synthesis Project sample. To update the systematic review of literature related to the effectiveness of interventions to reduce HCV seroconversion, we reperformed the automated searches and manually searched proceedings from scientific conferences in April 2010 to encompass the period after December 2006. We also examined bibliographies of qualitative reviews of $\mathrm{HCV}$ prevention to identify any additional studies [22-25]. Overlapping reports (ie, duplicate data from a single study) were identified based on matching study names, settings, and authors; this was followed by comparing sample sizes, years of data collection, and other study characteristics to select the most complete and informative report for our research question of interest. Eighteen reports from the pre-2007 set of studies and an additional 7 studies from the updated search were eligible for this analysis. All reports were in English, although the search included studies written in other languages.

We included studies in the current analysis if they reported HCV seroconversion rates or associations with
HCV seroconversion (odds ratio [OR], relative risk [RR], hazard ratio) in PWID in relation to interventions that could, in theory, prevent HCV infection by reducing unsafe injections. Thus, the scope of this analysis included studies of the association between HCV seroconversion and participation in drug-treatment programs, syringe-access programs (syringe exchange or distribution, or pharmacy sales), supervised injection facilities, syringe disinfection with bleach, individual behavioral interventions, or combinations of any of these services, all measured at the individual level. Studies conducted at the population level (eg, comparison of $\mathrm{HCV}$ infection rates pre- and postimplementation of syringeexchange programs) were not included, principally because none used seroconversion as the outcome measure. To our knowledge, no study has directly measured risk of sexual transmission of HCV in PWID or the effect of sexual risk reduction on HCV transmission. Given this and the much lower transmission efficiency via this route of exposure in other populations, prevention of sexual HCV transmission in PWID is currently of lesser importance, and thus this paper was restricted to prevention of injection-related transmission [26, 27].

Adjusted effect measures were used in the analysis where they were included in the source studies, under the assumption that adjustment was performed to remove bias in the estimate of the association between the intervention and risk of HCV seroconversion. Several cohort studies used ORs, including adjusted ORs, as their estimators; it has been shown that when the disease is common (incidence above 10 infections/100 PY), the OR will be biased away from the null value [28]. To correct for this bias, the ORs (crude or adjusted) and their 95\% confidence intervals (CIs) were transformed into relative risks and their respective CIs [28]. When ORs were calculated in case-control studies and incidence density sampling was used to select controls, no transformation was necessary [29]. In studies that did not report ORs, rate ratios, hazard ratios, relative risks, or risk ratios as a measure of the effect of the intervention on HCV seroconversion, an unadjusted relative risk was calculated using the incidence data provided. If the study reported cumulative incidence of HCV seroconversion in a cohort, the effect measure was calculated [28] and labeled a risk ratio; if the study reported incidence density of HCV seroconversion, the effect measure was labeled an incidence rate ratio. For 1 study [30] that reported only hazard ratios and $P$ values, CIs were calculated using standard methods [31]. Table 1 includes a column to show that risk measure was used in the analysis.

We evaluated heterogeneity of effects using $Q$ and $I^{2}$ statistics. We tested $Q$ values for significance using the $\chi^{2}$ distribution; we considered $I^{2}$ values $>50 \%$ to reflect meaningful heterogeneity [53]. We calculated summary estimates of the effects of similar interventions using Comprehensive Meta-Analysis software [54]. We also performed meta-analysis for subsets within intervention 


\begin{tabular}{|c|c|c|c|c|c|c|}
\hline $\begin{array}{l}\text { 1st author, } \\
\text { publication year }\end{array}$ & $\begin{array}{l}\text { Location and } \\
\text { study years }\end{array}$ & Design & $\begin{array}{l}\text { Sample size } \\
\text { and HCV } \\
\text { incidence rate } \\
(95 \% \mathrm{Cl})\end{array}$ & Intervention & $\begin{array}{c}\text { Published association with } \\
\text { HCV seroconversion }\end{array}$ & $\begin{array}{l}\text { Risk measure used in } \\
\text { analysis }(95 \% \mathrm{Cl})\end{array}$ \\
\hline \multicolumn{7}{|l|}{$\begin{array}{l}\text { 1a. Behavioral } \\
\text { interventions }\end{array}$} \\
\hline Garfein, 2007 [32] & $\begin{array}{l}\text { Multisite, } \\
\text { United States } \\
\text { 1999-2004 }\end{array}$ & RCT, Cohort & $\begin{array}{l}N=854 \\
18.4 / 100 \mathrm{PY} \\
(14.4-23.0)\end{array}$ & $\begin{array}{l}\text { Peer education (PEI) vs } \\
\text { equal-attention controls } \\
\text { (6 sessions each) }\end{array}$ & $\begin{array}{l}\text { OR PEI vs controls } 1.15 \\
(95 \% \mathrm{Cl}, .72-1.82)\end{array}$ & $\begin{array}{l}\text { Published unadjusted odds } \\
\text { ratio (PEI vs controls): } \\
\text { OR }=1.15(.72-1.82)\end{array}$ \\
\hline Stein, 2009 [33] & $\begin{array}{l}\text { Providence, RI, } \\
\text { United States } \\
\text { 2001-2004 }\end{array}$ & RCT, Cohort & $\begin{array}{l}N=89 \\
15.7 / 2 y\end{array}$ & $\begin{array}{l}\text { MI vs equal-attention } \\
\text { controls ( } 4 \text { sessions) }\end{array}$ & $\begin{array}{l}\text { Cumulative incidence } 18 \%(95 \% \mathrm{Cl} \text {, } \\
5.9-30 \%) \text { in } \mathrm{MI} \text { vs } 14 \% \text { in } \\
\text { controls (95\% Cl, 4.3-23.6\%) }\end{array}$ & $\begin{array}{l}\text { Calculated unadjusted risk } \\
\text { ratio (MI vs controls): } \\
\operatorname{RR}=1.28(.49-3.35)\end{array}$ \\
\hline \multicolumn{7}{|c|}{$\begin{array}{l}\text { 1b. Substance-use } \\
\text { treatment: Not specified }\end{array}$} \\
\hline Brunton, 2000 [34] & $\begin{array}{l}\text { Multisite, } \\
\text { New Zealand } \\
1994-1996\end{array}$ & OBS, Cohort & $\begin{array}{l}N=39 \\
23 \% / 2 y \\
(11 \%-30 \%)\end{array}$ & In treatment at follow-up & $\begin{array}{l}\text { Cumulative incidence } 50 \% \\
\text { in treatment }(7 / 14) \text { vs } 8 \% \\
\text { not in treatment }(2 / 25)\end{array}$ & $\begin{array}{l}\text { Calculated unadjusted } \\
\text { risk ratio: } \\
R R=6.25(1.3-30.09)\end{array}$ \\
\hline Lamothe, 1997 [35] & $\begin{array}{l}\text { Montreal, } \\
\text { Canada } \\
1992\end{array}$ & OBS, Cohort & $\begin{array}{l}N=63 \\
27.1 / 100 P Y \\
(18.0-29.1)\end{array}$ & In treatment at follow-up & $\begin{array}{l}\text { Incidence rate } 30.5 / 100 \text { PY } \\
\text { in treatment vs } 25.2 / 100 \\
\text { PY not in treatment }\end{array}$ & $\begin{array}{l}\text { Published unadjusted } \\
\text { hazard ratio: } \\
H R=1.02(.48-2.02)\end{array}$ \\
\hline Maher, 2006 [8] & $\begin{array}{l}\text { New South Wales, } \\
\text { Australia } \\
1999-2002\end{array}$ & OBS, Cohort & $\begin{array}{l}N=368 \\
30.8 / 100 \mathrm{PY} \\
(24.3-39.0)\end{array}$ & In treatment at enrollment & $\begin{array}{l}\text { Incidence rate } 28.9 / 100 \mathrm{PY} \\
\text { in treatment vs } 34.3 / 100 \\
\text { PY not in treatment }\end{array}$ & $\begin{array}{l}\text { Calculated unadjusted } \\
\text { incidence rate ratio: } \\
\text { IRR }=.84(.52-1.37)\end{array}$ \\
\hline Patrick, 2001 [36] & $\begin{array}{l}\text { Vancouver, } \\
\text { Canada } \\
\text { 1996-1999 }\end{array}$ & OBS, Cohort & $\begin{array}{l}N=155 \\
29.1 / 100 P Y \\
(22.3-37.3)\end{array}$ & $\begin{array}{l}\text { Nonmethadone addiction } \\
\text { treatment during follow-up }\end{array}$ & $\begin{array}{l}\text { Cumulative incidence } 66.7 \% \\
\text { in those in addiction therapy vs } \\
35.8 \% \text { in those not in treatment }\end{array}$ & $\begin{array}{l}\text { Calculated unadjusted } \\
\quad \text { risk ratio: } \\
R R=1.86(1.20-2.35)\end{array}$ \\
\hline Smyth, 2003 [14] & $\begin{array}{l}\text { Dublin, Ireland } \\
\text { 1992-1998 }\end{array}$ & OBS, Cohort & $\begin{array}{l}N=100 \\
66 / 100 P Y \\
(51-84)\end{array}$ & $\begin{array}{l}\text { Addiction treatment }>3 \text { mo } \\
\text { vs less during follow-up }\end{array}$ & $\begin{array}{l}\text { Incidence rate } 52 / 100 \mathrm{PY} \text { among } \\
\text { those in treatment }>3 \text { mo vs } \\
75 / 100 \mathrm{PY} \text { for others, } P=.16\end{array}$ & $\begin{array}{l}\text { Calculated unadjusted } \\
\text { incidence rate ratio: } \\
\text { IRR }=.69(.42-1.1)\end{array}$ \\
\hline \multicolumn{7}{|c|}{$\begin{array}{l}\text { 1c. Substance-use treatment: } \\
\text { opiate-replacement therapy }\end{array}$} \\
\hline Craine, 2009 [37] & $\begin{array}{l}\text { South Wales, } \\
\text { United Kingdom } \\
\text { 2004-2006 }\end{array}$ & OBS, Cohort & $\begin{array}{l}N=286 \\
5.9 / 100 P Y \\
(3.4-9.5)\end{array}$ & In ORT at follow-up & $\begin{array}{l}\text { Incidence rate } 2.9 / 100 \mathrm{PY} \text { in those } \\
\text { in ORT vs } 10.6 / 100 \mathrm{PY} \text { in others }\end{array}$ & $\begin{array}{l}\text { Published adjusted } \\
\text { incidence rate ratio: } \\
\text { AIRR }=.34(.12-.99)\end{array}$ \\
\hline Crofts, 1997 [38] & $\begin{array}{l}\text { Victoria, Australia } \\
\text { 1991-1995 }\end{array}$ & $\begin{array}{l}\text { OBS, } \\
\text { Retrospective } \\
\text { cohort }\end{array}$ & $\begin{array}{l}N=73 \\
22.2 / 100 \mathrm{PY} \\
(14.2-34.8)\end{array}$ & $\begin{array}{l}\text { Continuous vs interrupted } \\
\text { or no ORT during follow-up }\end{array}$ & $\begin{array}{l}\text { Cumulative incidence } 36.9 \% \\
\text { continuous ORT vs } 14.2 \% \\
\text { interrupted vs } 21.4 \% \text { no ORT }\end{array}$ & $\begin{array}{l}\text { Calculated unadjusted } \\
\text { risk ratio (Continuous } \\
\text { ORT vs interrupted } \\
\text { or no ORT): } \\
\text { RR }=2.25(.91-5.54)\end{array}$ \\
\hline Dolan, 2005 [39] & $\begin{array}{l}\text { New South Wales, } \\
\text { Australia } \\
1998-2002\end{array}$ & $\begin{array}{l}\text { RCT, } \\
\text { Retrospective } \\
\text { cohort }\end{array}$ & $\begin{array}{l}N=39 \\
21.3 / 100 P Y \\
(15.6-29.2)\end{array}$ & $\begin{array}{l}\text { Random assignment to ORT } \\
\text { vs control while in prison }\end{array}$ & $\begin{array}{l}\text { Incidence rate } 16 / 100 \mathrm{PY} \text { in ORT } \\
\text { treatment group vs } 27 / 100 \\
\mathrm{PY} \text { in controls }\end{array}$ & $\begin{array}{l}\text { Published adjusted } \\
\text { hazard ratio: } \\
\text { AHR }=.5(.24-1.11)\end{array}$ \\
\hline Hallinan, 2004 [40] & $\begin{array}{l}\text { Sydney, Australia } \\
\text { 1996-2003 }\end{array}$ & $\begin{array}{l}\text { OBS, } \\
\text { Retrospective } \\
\text { cohort }\end{array}$ & $\begin{array}{l}N=54 \\
3.8 / 100 P Y \\
(1.2-8.9)\end{array}$ & Continuous vs interrupted ORT & $\begin{array}{l}\text { Incidence rate } 1.3 / 100 \text { PY } \\
\text { continuous ORT vs } 7.4 / 100 \\
\text { PY interrupted ORT }\end{array}$ & $\begin{array}{l}\text { Calculated unadjusted } \\
\text { incidence rate ratio } \\
\text { (continuous vs } \\
\text { interrupted ORT): } \\
\text { IRR }=.18(.02-1.59 \text { ) }\end{array}$ \\
\hline Lucidarme, 2004 [7] & $\begin{array}{l}\text { Multisite, France } \\
\text { 1999-2000 }\end{array}$ & OBS, Cohort & $\begin{array}{l}N=165 \\
9 / 100 P Y \\
(4.6-13.4)\end{array}$ & $\begin{array}{l}\text { Substitution treatment } \\
\text { at enrollment }\end{array}$ & $\begin{array}{l}\text { Incidence rate } 7.7 / 100 \mathrm{PY} \text { those } \\
\text { in treatment vs } 14.1 / 100 \\
\text { PY not in treatment }\end{array}$ & $\begin{array}{l}\text { Published adjusted } \\
\text { hazard rate: } \\
\text { AHR }=.41(.12-1.40)\end{array}$ \\
\hline Rezza, 1996 [41] & $\begin{array}{l}\text { Naples, Italy } \\
1991-1993\end{array}$ & $\begin{array}{l}\text { OBS, Nested } \\
\text { case-control }\end{array}$ & $\begin{array}{l}N=106 \\
28.6 / 100 \mathrm{PY} \\
(17.8-43.4)\end{array}$ & ORT during follow-up & $\begin{array}{l}21.2 \% \text { of seroconverters vs } 28.2 \% \\
\text { of controls in ORT during follow-up }\end{array}$ & $\begin{array}{l}\text { Calculated adjusted } \\
\text { relative risk: } \\
\mathrm{ARR}=.42(.14-1.08)\end{array}$ \\
\hline
\end{tabular}




\begin{tabular}{|c|c|c|c|c|c|c|}
\hline $\begin{array}{l}\text { 1st author, } \\
\text { publication year }\end{array}$ & $\begin{array}{l}\text { Location and } \\
\text { study years }\end{array}$ & Design & $\begin{array}{l}\text { Sample size } \\
\text { and HCV } \\
\text { incidence rate } \\
(95 \% \mathrm{Cl})\end{array}$ & Intervention & $\begin{array}{l}\text { Published association with } \\
\text { HCV seroconversion }\end{array}$ & $\begin{array}{l}\text { Risk measure used in } \\
\text { analysis }(95 \% \mathrm{Cl})\end{array}$ \\
\hline Thiede, 2000 [42] & $\begin{array}{l}\text { Seattle, WA, } \\
\text { United States } \\
\text { 1994-1998 }\end{array}$ & OBS, Cohort & $\begin{array}{l}N=78 \\
9.0 \% / y \\
(5.7-12.2)\end{array}$ & $\begin{array}{l}\text { Continued vs disrupted or left } \\
\text { ORT during follow-up }\end{array}$ & 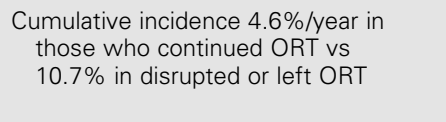 & $\begin{array}{l}\text { Calculated unadjusted } \\
\text { risk ratio (continued } \\
\text { vs disrupted or left ORT): } \\
\mathrm{RR}=.42(.05-2.76)\end{array}$ \\
\hline van Beek, 1998 [43] & $\begin{array}{l}\text { Sydney, Australia } \\
\text { 1992-1995 }\end{array}$ & $\begin{array}{l}\text { OBS, } \\
\text { Retrospective } \\
\text { cohort }\end{array}$ & $\begin{array}{l}N=152 \\
20.9 / 100 \mathrm{PY} \\
(13.5-28.3)\end{array}$ & Ever in ORT vs never in ORT & $\begin{array}{l}\text { Incidence rate 18.0/100 } \\
\text { PY both groups }\end{array}$ & $\begin{array}{l}\text { Calculated unadjusted } \\
\text { incidence rate ratio: } \\
\operatorname{IRR}=1.0(.40-2.49)\end{array}$ \\
\hline \multicolumn{7}{|c|}{$\begin{array}{l}\text { 1d. Syringe/injecting } \\
\text { equipment access programs }\end{array}$} \\
\hline Hagan, 1995 [44] & $\begin{array}{l}\text { Tacoma, WA, } \\
\text { United States } \\
\text { 1991-93 }\end{array}$ & OBS, Case-control & $\begin{array}{l}N=46 \\
\text { Incidence not } \\
\text { applicable }\end{array}$ & Ever vs never participate in SEP & $\begin{array}{l}\text { AOR .14 (95\% Cl .03-.62) } \\
\text { SEP users vs nonusers }\end{array}$ & $\begin{array}{l}\text { Published adjusted OR: } \\
\text { AOR }=.14(.03-.62)\end{array}$ \\
\hline Hagan, 2004 [45] & $\begin{array}{l}\text { Seattle, WA, } \\
\text { United States } \\
\text { 1994-2001 }\end{array}$ & OBS, Cohort & $\begin{array}{l}N=484 \\
11.6 / 100 \mathrm{PY} \\
(9.8-13.5)\end{array}$ & Any use of SEP during follow-up & HR 1.4 SEP users vs nonusers. & $\begin{array}{l}\text { Published unadjusted } \\
\text { hazard ratio: } \\
\text { HR }=1.4(.9-1.9)\end{array}$ \\
\hline Holtzman, 2009 [46] & $\begin{array}{l}\text { Multisite, } \\
\text { United States } \\
\text { 1994-2004 }\end{array}$ & OBS, Cohort & $\begin{array}{l}N=1288 \\
\text { Incidence } \\
\text { not given }\end{array}$ & Any use of SEP during follow-up & $\begin{array}{l}\text { AOR } 1.49 \text { (95\% Cl .96-2.29) } \\
\text { SEP users vs nonusers }\end{array}$ & $\begin{array}{l}\text { Calculated adjusted } \\
\text { relative risk: } \\
\text { ARR }=1.41(.96-2.01)\end{array}$ \\
\hline Lamothe, 1997 [35] & $\begin{array}{l}\text { Montreal, } \\
\text { Canada } \\
1992\end{array}$ & OBS, Cohort & $\begin{array}{l}N=63 \\
27.1 / 100 \mathrm{PY} \\
(18.0-29.1)\end{array}$ & $\begin{array}{l}\text { Obtained any needles from } \\
\text { SEP during follow-up }\end{array}$ & $\begin{array}{l}\text { 55.9/100 PY SEP users vs } \\
\text { 31.0/100 PY in nonusers. }\end{array}$ & $\begin{array}{l}\text { Published unadjusted } \\
\text { hazard ratio: } \\
H R=2.24(1.01-4.98)\end{array}$ \\
\hline Patrick, 2001 [36] & $\begin{array}{l}\text { Vancouver, } \\
\text { Canada } \\
\text { 1996-1999 }\end{array}$ & OBS, Cohort & $\begin{array}{l}N=155 \\
29.1 / 100 \mathrm{PY} \\
(22.3-37.3)\end{array}$ & $\begin{array}{l}\text { Frequent SEP attendance } \\
\text { ( }>1 \text { time/week) during follow-up }\end{array}$ & $\begin{array}{l}\text { Cumulative incidence } 54.7 \% / y e a r \\
\text { frequent attenders vs } 26.3 \% \text { others }\end{array}$ & $\begin{array}{l}\text { Published adjusted } \\
\text { hazard ratio: } \\
\text { AHR }=2.56(1.37-4.79)\end{array}$ \\
\hline Roy, 2007 [30] & $\begin{array}{l}\text { Montreal, } \\
\text { Canada } \\
\text { 1997-2003 }\end{array}$ & OBS, Cohort & $\begin{array}{l}N=543 \\
27.1 / 100 \mathrm{PY} \\
(23.4-30.9)\end{array}$ & Any use of SEP during follow-up & $\begin{array}{l}\text { HR } 3.02 \text { SEP users vs } \\
\text { nonusers, } P=.18 .\end{array}$ & $\begin{array}{l}\text { Calculated }{ }^{a} \text { unadjusted } \\
\text { hazard ratio: } \\
\text { HR = } 3.02 \text { (2.32-3.72) }\end{array}$ \\
\hline Thorpe, 2002 [47] & $\begin{array}{l}\text { Chicago, IL, } \\
\text { United States } \\
\text { 1997-1999 }\end{array}$ & OBS, Cohort & $\begin{array}{l}N=353 \\
10 / 100 P Y \\
(6.7-14.4)\end{array}$ & Any use of SEP during follow-up & HR 1.29 SEP users vs nonusers. & $\begin{array}{l}\text { Published unadjusted } \\
\text { hazard ratio: } \\
H R=1.29(.6-2.79)\end{array}$ \\
\hline \multicolumn{7}{|c|}{ 1e. Syringe disinfection with bleach } \\
\hline Hagan, 2003 [48] & $\begin{array}{l}\text { Seattle, WA, } \\
\text { United States } \\
\text { 1994-2001 }\end{array}$ & $\begin{array}{l}\text { OBS, Nested } \\
\text { Case-control }\end{array}$ & $\begin{array}{l}N=195 \\
23.1 \% \\
(17.1-28.9)\end{array}$ & $\begin{array}{l}\text { Always vs <always used bleach } \\
\text { to disinfect used syringes } \\
\text { during follow-up }\end{array}$ & $\begin{array}{l}\text { Cumulative incidence } 26 \% / \text { year } \\
\text { among those who always bleached } \\
\text { vs } 22 \% \text { in those who did not } \\
\text { AOR } 1.4(95 \% \mathrm{Cl} .7-3.0) \text { always } \\
\text { used bleach vs others. }\end{array}$ & $\begin{array}{l}\text { Calculated adjusted } \\
\text { relative risk: } \\
\text { ARR }=1.21(.68-1.95)\end{array}$ \\
\hline Hagan, 2010 [49] & $\begin{array}{l}\text { Multisite, } \\
\text { United States } \\
\text { 1995-2000 }\end{array}$ & OBS, Cohort & $\begin{array}{l}N=483 \\
17.2 / 100 \mathrm{PY} \\
(13.2-22.4)\end{array}$ & $\begin{array}{l}\text { Always vs did not } \\
\text { always bleach shared } \\
\text { syringes during follow-up }\end{array}$ & $\begin{array}{c}\text { AOR } 1.14(95 \% \mathrm{Cl} .62-5.88) \text { shared } \\
\text { but always bleached vs others }\end{array}$ & $\begin{array}{l}\text { Calculated }^{\mathrm{b}} \text { adjusted } \\
\text { hazard ratio: } \\
\mathrm{AHR}=1.97(.29-2.45)\end{array}$ \\
\hline Hahn, 2002 [50] & $\begin{array}{l}\text { San Francisco, WA, } \\
\text { United States } \\
\text { 2000-2001 }\end{array}$ & OBS, Cohort & $\begin{array}{l}N=195 \\
25 / 1 / 100 \mathrm{PY} \\
(18.7-32.9)\end{array}$ & $\begin{array}{l}\text { Bleached all borrowed syringes } \\
\text { during follow-up }\end{array}$ & $\begin{array}{l}\text { Incidence rate } 38.5 / 100 \mathrm{PY} \text { bleached } \\
\text { all syringes vs } 46.5 / 100 \mathrm{PY} \text { in others. }\end{array}$ & $\begin{array}{l}\text { Published unadjusted } \\
\text { hazard ratio: } \\
\mathrm{HR}=.8(.3-2.2)\end{array}$ \\
\hline Kapadia, 2002 [51] & $\begin{array}{l}\text { Multisite, } \\
\text { United States } \\
\text { 1997-1999 }\end{array}$ & $\begin{array}{l}\text { OBS, Nested } \\
\text { case-control }\end{array}$ & $\begin{array}{l}N=468 \\
\text { Incidence } \\
\text { not given }\end{array}$ & $\begin{array}{l}\text { Always vs not always bleach } \\
\text { syringes during follow-up }\end{array}$ & $\begin{array}{l}\text { AOR } .45 \text { shared but always } \\
\text { bleached vs < all the time }\end{array}$ & $\begin{array}{l}\text { Published adjusted } \\
\text { odds ratio: } \\
\text { AOR }=.45(.11-1.55)\end{array}$ \\
\hline
\end{tabular}




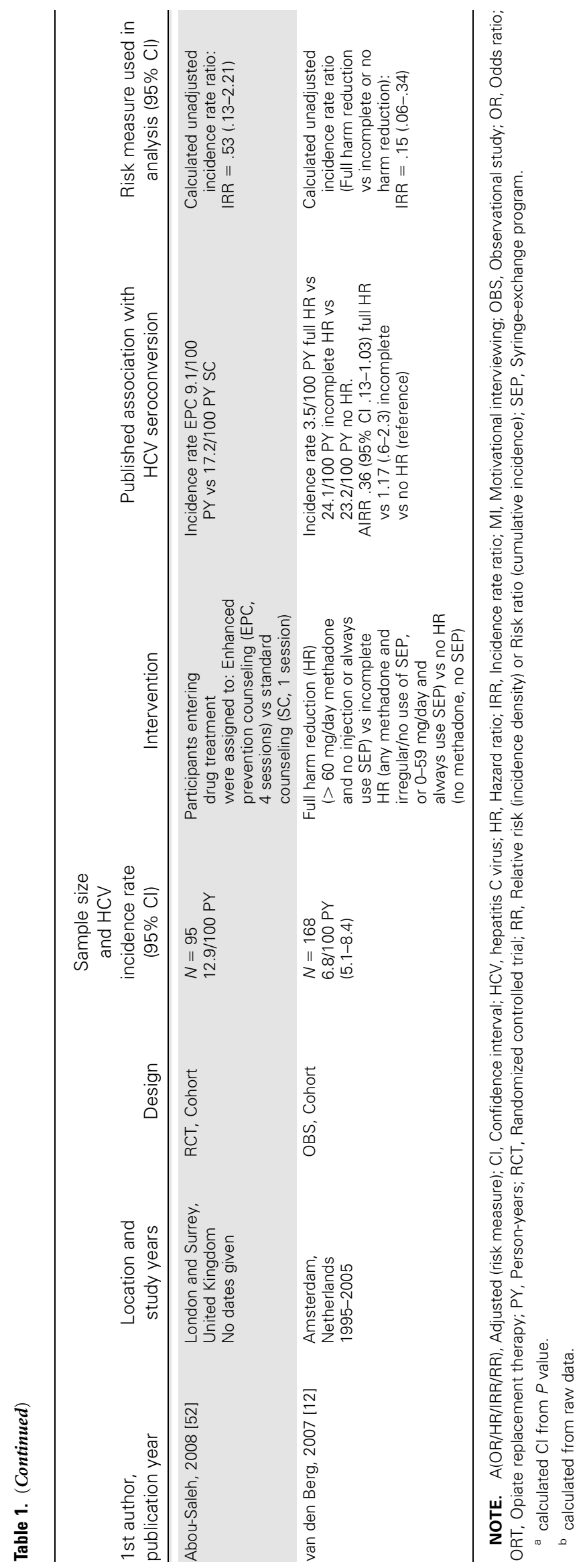

type defined by similarity in dose or comparison group. We used random effects models throughout to more accurately account for unmeasured sources of variation among studies [55]. We examined asymmetry in funnel plots of treatment effects against standard errors to assess potential publication bias [56].

\section{RESULTS}

A total of 26 studies were eligible for inclusion in this analysis $[7,8,12,14,30,32-52]$. Of these, 1 study was unpublished [35], and 2 studies $[35,36]$ reported on $>1$ intervention. The studies included 4 randomized controlled trials (RCTs) [32, 33, 39, 52]; the remainder were observational studies. The RCTs examined the effects of single interventions [32, 33, 39] or those provided in combination [52]. There were 4 case-control studies, 3 of which were nested in longitudinal designs [41, 44, 49, 51]. All other studies used longitudinal cohort designs.

As shown in Table 1 , most studies $(n=13)$ reported on the relation between substance-use treatment and HCV seroconversion; 5 of these studies did not specify the type of drug treatment measured, and 8 studies examined the effects of opiate-replacement therapy (ORT). In all, 7 studies reported on the association between syringe-access programs and HCV incidence; each of these examined needle-exchange programs. There were 4 reports on the effect of bleach disinfectant on HCV acquisition; 2 studies examined the association between multicomponent interventions and HCV seroconversion [12, 52]. No study reported on HCV seroconversion in relation to supervised injection facilities or pharmacy sales of syringes. All studies were from high-income countries in North America, Western Europe, or Asia. Most studies were completed before 2000. Funnel plots of effects by intervention type showed little evidence of publication bias (data not shown).

\section{Behavioral Interventions}

Of all the studies analyzed, 2 studies examined the effect of a behavioral intervention on the incidence of HCV infection in PWID (Tables 1a, 2a). The OR for the association between participation in a 6-session peer education training and $\mathrm{HCV}$ seroconversion was 1.15 (95\% CI, .72-1.82) indicating no difference between the intervention arm and attention-matched controls [32]. A different study assigned 89 individuals enrolled in drug treatment to 4 sessions of motivational interviewing or to an equal-attention control condition; 2-year cumulative HCV incidence was $18 \%$ in the motivational-interviewing group vs $14 \%$ in controls $(P=.6)$ [33]. The pooled relative risk was 1.18 (95\% CI, .76-1.81). The $Q$ statistic was not statistically significant, and $I^{2}$ was $0 \%$.

\section{Substance-Use Treatment: Not Specified}

In these studies, substance-use treatment could conceivably have represented a range of modalities, including attending self-help 
Table 2. Meta-Analyses of the Effect of Interventions on HCV Seroconversion in PWID.

$1^{\text {st }}$ author, publication year random effects estimate \& heterogeneity statistics

Relative risk $\left(95 \% \mathrm{Cl}^{1}\right)$

Forest plot

$\begin{array}{ll}\text { 2a. Behavioral interventions } & \\ \text { Garfein, } 2007 \text { (32) } & 1.15(0.72,1.82)^{2} \\ \text { Stein, } 2009 \text { (33) } & 1.34(0.43,4.20)^{2} \\ \quad \text { Random effects estimate } & 1.18(0.77,1.81)^{2}\end{array}$

$\mathrm{Q}, \mathrm{I}^{2}$

$0.6,0 \%$

2b. Substance-use treatment, not specified

\begin{tabular}{ll} 
Brunton $2000(34)$ & $6.25(1.30,30.09)$ \\
Lamothe $1997(35)$ & $1.02(0.48,2.02)$ \\
Maher 2006 (8) & $0.84(0.52,1.37)$ \\
Patrick 2001 (36) & $1.86(1.20,2.35)$ \\
Smyth $2003(14)$ & $0.69(0.42,1.15)$ \\
\multicolumn{1}{r}{ Random effects estimate } & $1.21(0.71,2.08)$ \\
\hline Q, I & $17.6^{* *}, 77 \%$
\end{tabular}

2c. Substance-use treatment, Opiate-Replacement Therapy (ORT)

Craine 2009 (37)

Crofts 1997 (38)

Dolan 2005 (39)

Hallinan 2004 (40)

Lucidarme 2004 (7)

Rezza 1996 (41)

Theide 2000 (42)

van Beek 1998 (43)

Random effects estimate

$\mathrm{Q}, \mathrm{I}^{2}$

$0.34(0.12,0.99)$

$2.25(0.91,5.54)$

$0.50(0.24,1.11)$

$0.18(0.02,1.59)$

$0.41(0.12,1.40)$

$0.42(0.14,1.08)$

$0.42(0.05,2.76)$

$1.00(0.40,2.49)$

$0.60(0.35,1.03)$

$12.8,45 \%$

2d. Syringe access program, syringe exchange

Hagan 1995 (44)

$0.14(0.03,0.62)$

Hagan 2004 (45)

$1.40(0.90,1.90)$

Holtzman 2009 (46)

$1.41(0.96,2.01)$

Lamothe 1997 (35)

$2.24(1.01,4.98)$

Patrick 2001 (36)

$2.56(1.37,4.79)$

Roy 2007 (30)

$3.02(2.32,3.72)$

Thorpe 2002 (47)

$1.29(0.60,2.79)$

Random effects estimate

$1.62(1.04,2.52)$

$\mathrm{Q}, \mathrm{I}^{2}$

$$
32.3^{* *}, 81 \%
$$

\begin{tabular}{|c|c|}
\hline 2e. Syringe disinfection, bleach & \\
\hline Hagan 2003 (48) & $1.21(0.68,1.95)$ \\
\hline Hagan 2010 (49) & $1.97(0.54,7.14)$ \\
\hline Hahn 2002 (50) & $0.80(0.30,2.20)$ \\
\hline Kapadia 2002 (51) & $0.45(0.11,1.55)$ \\
\hline Random effects estimate & $1.07(0.70,1.63)$ \\
\hline$Q, I^{2}$ & $3.0,1 \%$ \\
\hline
\end{tabular}

2f. Multicomponent interventions 


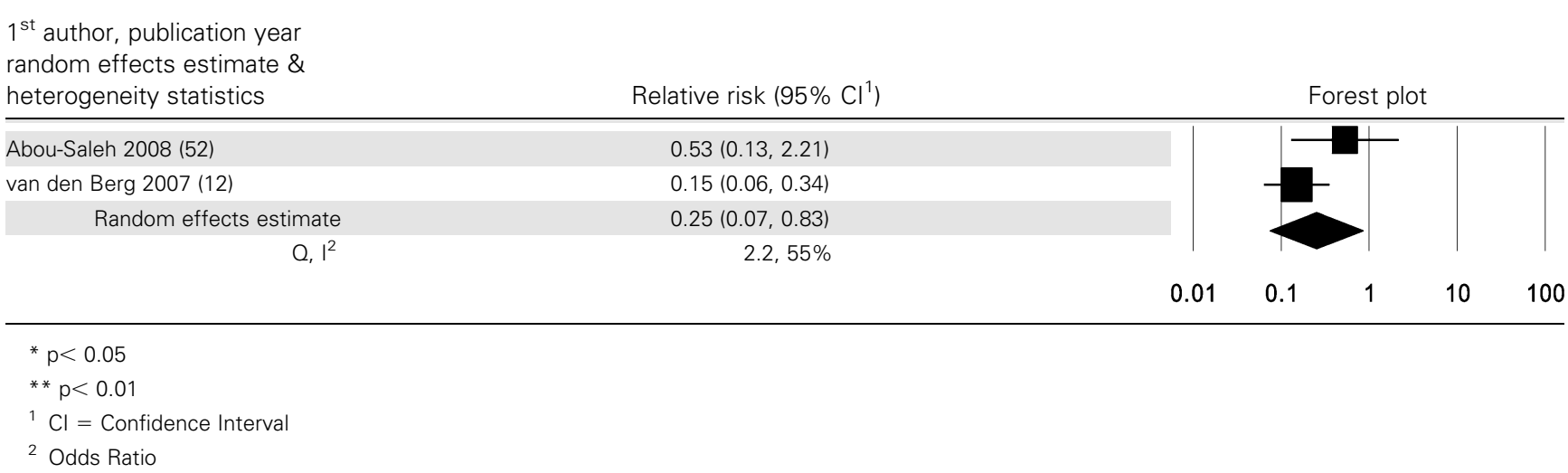

groups, outpatient drug-free programs, or inpatient residential programs. Timing and duration of exposure to substance-use treatment was measured in a number of ways, including whether participants were in treatment at study enrollment, were in treatment at the end of the follow-up period, or received treatment throughout follow-up or for a specified length of time (Table 1b). As shown in Table 2b, there was substantial heterogeneity in the association between participation in these programs and HCV seroconversion, with 2 studies showing lower rates of $\mathrm{HCV}$ infection among those exposed to drug treatment $[8,14]$ and 2 showing statistically significant higher rates $[34,36]$. HCV incidence rates in the studies ranged from cumulative incidence of $23 \%$ over a 2 -year period [34] to 66 infections per $100 \mathrm{PY}$ [14]. The $Q$ value was statistically significant and $I^{2}$ was $77 \%$, so the pooled relative risk $(1.21 ; 95 \%$ CI, .71-2.08) should be interpreted with caution. Excluding the study that compared substance-use treatment to a comparison group of individuals who received treatment for a shorter period of time [14], the pooled relative risk was 1.43, (95\% CI, .79-2.58); the $Q$ value was not significant $(P=.24)$ and $I^{2}=73 \%$.

\section{Substance-Use Treatment: Opiate-Replacement Therapy}

In the 8 studies evaluating the effect of ORT on HCV incidence, degree of exposure was measured for whether participants were in ORT at study enrollment or at the end of the follow-up period, or had remained in treatment throughout the followup period (Table 1c). In the RCT that evaluated the effect of ORT started when participants were incarcerated, incidence was measured 1-5 years after the end of trial, when some participants were still in prison and others had been released [39].

In several of these studies, HCV incidence rates among participants in treatment were rather low, including 2.9/100 PY among PWID in the United Kingdom who were in ORT at the end of follow-up [37]; 1.3/100 PY among Sydney PWID who remained in treatment throughout [40]; and cumulative incidence of $4.6 \%$ at the end of 1 year in PWID who remained in treatment in Seattle [42]. In 1 early study, HCV incidence was higher among those who remained in ORT as compared with that among those who left or had no ORT during follow-up [38].

As shown in Table 2c, the pooled relative risk of HCV seroconversion in relation to ORT was .60 (95\% CI, .35-1.03). Heterogeneity was not significant and $I^{2}$ was $45 \%$. A pooled relative risk was also calculated for studies where the comparison group excluded "interrupted" ORT [7, 37, 39, 41, 43]; the pooled RR estimate was similar to that for the full set of ORT studies ( RR, .52; 95\% CI, .34-.79), but there was much less heterogeneity, with $Q=2.9(P=.57)$ and $I^{2}=0 \%$. When the analysis was restricted to studies that compared continuous enrollment in ORT (throughout follow-up) to those who disrupted or left ORT $[38,40,42]$, the pooled RR was .70, $(95 \%$ CI, .14-3.60), the $Q$ was $5.8(P=.056)$, and $I^{2}$ was $65 \%$.

\section{Syringe-Access Programs}

All 7 studies that examined participation in syringe-access programs (syringe-exchange programs [SEP]) in relation to HCV seroconversion were from North America (Table 1d). Sample sizes ranged from 46 to 1288 participants, and median sample size was 353 participants. All but 1 study [36] examined SEP exposure as any use compared with no use during the period of susceptibility to $\mathrm{HCV}$ infection.

Only 1 study, which used a case-control design [44], found that participation in an SEP was associated with a significantly lower risk of HCV seroconversion. A Canadian study [36] showed significantly elevated risk of HCV infection in frequent exchange-program attendees compared with the risk in other PWID. All other studies reported no significant association. The resulting pooled effect (RR, 1.62; 95\% CI, 1.04-2.52) showed substantial heterogeneity $\left(Q=32.3 ; P<.01 ; I^{2}=81 \%\right)$.

\section{Syringe Disinfection}

The effect on risk of HCV infection of disinfecting syringes with bleach was evaluated in 4 studies in the United States [Table 1e]. 
All studies defined bleach use as either always using bleach or disinfecting all syringes with bleach. The estimates of the association ranged between .42 and 1.97, and the pooled estimate was 1.08 (95\% CI, .66-1.75). Heterogeneity was not significant.

\section{Multicomponent Programs}

In 2 studies, investigators examined the effect of participation in multicomponent interventions [Table 1f]. In the United Kingdom study, ORT combined with enhanced HCV prevention counseling was compared with ORT alone; HCV seroconversion was lower among those in the combined intervention group (9.1/100 PY vs 17.2/100 PY in the ORT alone group; $P>$.05) [52]. In the Amsterdam study, "full participation in harm reduction"-_efined as $>60 \mathrm{mg}$ methadone per day and always using SEP-was compared with "less than full harm reduction" or no harm reduction [12]. HCV incidence was 3.5/100 PY in the full-harm-reduction group compared with 23.9/100 PY in other study participants. As shown in Table 2f, the pooled RR was .25 (95\% CI, .07-.83). Although the $Q$ value was not significant, $I^{2}$ was $55 \%$.

\section{DISCUSSION}

The meta-analysis found a substantial and statistically significant reduction in HCV incidence in PWID-of approximately $75 \%$ - when combination prevention strategies were applied. This finding is consistent with an understanding that an array of factors facilitate HCV transmission among PWID, including the large disease reservoir of $\mathrm{HCV}$-infectious injectors, the efficiency with which HCV may be transmitted via a number of different drug injection-related practices, and the chaotic and rushed atmosphere of the injection setting $[9,10,47,50,57]$. Thus, multicomponent interventions that support a range of strategies (reduction or elimination of drug injection, adoption of safe injection practices through the provision of sterile syringes and drug-preparation equipment, or behavior-change counseling) would be expected to achieve greater success than those offering fewer options for lowering risk.

Both of the multicomponent interventions examined here included ORT; in the Abou-Saleh study, reduced HCV incidence was observed when enhanced prevention counseling was administered to PWID while in substitution treatment, and in the van den Berg study, lowest HCV incidence was among those receiving $\geq 60 \mathrm{mg}$ of methadone per day and obtaining all their syringes from an SEP [12, 52]. However, the meta-analysis of ORT alone showed a less substantial, inconsistent impact on HCV seroconversion risk. Therefore, one cannot conclude that the large-magnitude effect of multicomponent programming on HCV infection rates is wholly attributable to ORT. In several studies of ORT, remaining in treatment was associated with significant reductions in injection frequency, but not elimination $[12,37,40]$. Thus, maintaining control over one's drug intake via injection may be a key element in reducing HCV risk.

A strength of this analysis is its restriction to studies that used $\mathrm{HCV}$ seroconversion as an outcome measure, as opposed to $\mathrm{HCV}$ prevalence or injection risk behavior. Although scientifically defensible, this restriction left a relatively small number of studies. The results of the meta-analysis are also consistent with the conclusion of qualitative reviews of HCV prevention in PWID, that packages of harm reduction programs may be effective [22-25]. Another meta-analysis from the HCV Synthesis Project showed that the expansion of syringe access and ORT programs in high-income countries was associated with a lengthening in the time from onset of drug injection to acquisition of HCV [2]. The results shown here are also consistent with the findings of a qualitative study of long-term HCVseronegative PWID who reported that they used a combination of strategies to avoid withdrawal symptoms and practice safe injection [58].

The study has limitations that must be kept in mind in interpreting the results. As with all meta-analyses, we were restricted to the data that could be obtained from written reports. In some cases, information bias may have been introduced by the use of antibody tests to detect seroconversion or the use of self-report to assess intervention participation; such error (if nondifferential) would have biased results toward a null association. Null and weak effects in some studies may also have been observed as a result of low dose of exposure to the intervention in question. Indeed, hypothesis testing should be led by an assertion of the degree of exposure to the intervention that is likely to prevent transmission. For example, being in substance-use treatment on the date of study enrollment or on the follow-up visit may represent sporadic treatment. However, focusing on studies where ORT was received throughout follow-up $(\mathrm{RR}=.71)$ did not reveal a larger-magnitude effect ( $\mathrm{RR}=.60$ for the full set of ORT studies). In contrast, measurement of syringe disinfection with bleach was consistent across the 4 studies (bleached all syringes during follow-up), so the finding of a distribution of RRs around the null value of the pooled estimate $(\mathrm{RR}=1.07, P>0.05)$ rather strongly supports a conclusion of no effect on HCV transmission.

Removal of confounding cannot be assumed in the analysis shown here. Even adjusted RRs may not have been calculated following data-based approaches [59], and multivariate models may have included only statistically significant terms and thus omitted important confounding factors [29, 60]. Further, structural interventions such as expanded syringe access are designed to reduce risk of infection for a population of PWID; research has shown that individual-level comparisons of SEP users to nonusers may be particularly prone to volunteer bias (a form of confounding) in that exchange programs attract and retain higher-risk PWID [61, 62] Consequently, the positive individual association between HCV acquisition and SEP 
participation found in this meta-analysis should not be interpreted as suggesting that SEP participation increases risk of HCV acquisition. Less biased evaluations of SEPs would require random assignment of communities; ethical considerations, research cost, and possible community resistance to being assigned to a non-SEP control condition all make such community-level evaluations unlikely in the foreseeable future. The variation in HCV incidence rates among PWID noted in the introduction also suggests that many communities would be needed in a community-level controlled trial. A final comment on the limitations is that the small number of studies by intervention type limited our ability to detect publication bias; pooled effect estimates in this study may thus overestimate or underestimate the true effects. The small sample size also precluded subgroup analyses in relation to PWID characteristics.

There are several implications for future research on $\mathrm{HCV}$ prevention for PWID, both for content and design. Foremost is that multicomponent interventions that include methods to reduce drug-use frequency and to support safe injection should be developed and tested in a variety of settings and subsets of PWID (eg, young, racial or ethnic minority PWID, and low- and middle-income countries). Design of an evaluation should be based on an a priori conceptualization of what constitutes an adequate dose of the intervention and assuring that measurement of exposure reflects dosage. Reporting of the results of future evaluations should include detailed descriptions of the intervention for dose and duration, and examine the effects of participation in multiple intervention types. In the meantime, the currently available research indicates that HCV can be prevented among PWID.

\section{Funding}

This study was supported by the United States National Institute on Drug Abuse (R01 DA018609); and the National Institutes of Health-funded Center for Drug Use and HIV Research (P30 DA121041).

\section{References}

1. Gerberding JL. Incidence and prevalence of human immunodeficiency virus, hepatitis $\mathrm{B}$ virus, hepatitis $\mathrm{C}$ virus, and cytomegalovirus among health care personnel at risk for blood exposure: Final report from a longitudinal study. J Infect Dis 1994; 170:1410-7.

2. Hagan H, Pouget ER, Des Jarais DC, Lelutiu-Weinberger C. Metaregression of hepatitis $\mathrm{C}$ virus infection in relation to time since onset of illicit drug injection: The influence of time and place. Am J Epidemiol 2008; 168:1099-109.

3. Eicher AD, Crofts N, Benjamin S, Deutschmann P, Rodger AJ. A certain fate: Spread of HIV among young injecting drug users in Manipur, north-east India. AIDS Care 2000; 12:497-504.

4. Kuo I, ul-Hasan S, Galai N, et al. High HCV seroprevalence and HIV drug use risk behaviors among injection drug users in Pakistan. Harm Reduct J 2006; 3:26.

5. van de Laar TJ, Langendam MW, Bruisten SM, et al. Changes in risk behavior and dynamics of hepatitis $\mathrm{C}$ virus infections among young drug users in Amsterdam, the Netherlands. J Med Virol 2005; 77:509-18.
6. van den Berg CH, Smit C, Bakker M, et al. Major decline of hepatitis $\mathrm{C}$ virus incidence rate over two decades in a cohort of drug users. Eur J Epidemiol 2007; 22:183-93.

7. Lucidarme D, Bruandet A, Ilef D, et al. Incidence and risk factors of HCV and HIV infections in a cohort of intravenous drug users in the North and East of France. Epidemiol Infect 2004; 132:699-708.

8. Maher L, Jalaludin B, Chant KG, et al. Incidence and risk factors for hepatitis $\mathrm{C}$ seroconversion in injecting drug users in Australia. Addiction 2006; 101:1499-508.

9. Hagan $\mathrm{H}$, Pouget ER, Williams IT, et al. Attribution of hepatitis $\mathrm{C}$ virus seroconversion risk in young injection drug users in 5 US cities. J Infect Dis 2010; 201:378-85.

10. Hagan $\mathrm{H}$, Theide $\mathrm{H}$, Weiss NS, et al. Sharing of drug preparation equipment as a risk factor for hepatitis C. Am J Public Health 2001; 91:42-6.

11. Garten RJ, Lai S, Zhang J, et al. Rapid transmission of hepatitis $C$ virus among young injecting heroin users in southern China. Int J Epidemiol 2004; 33:182-8.

12. van den Berg C, Smit C, Van Brussel G, et al. Full participation in harm reduction programmes is associated with decreased risk for human immunodeficiency virus and hepatitis $\mathrm{C}$ virus: Evidence from the Amsterdam Cohort Studies among drug users. Addiction 2007; 102:1454-62.

13. Maher L, Li J, Jalaludin B, Chnat KG, Kaldor JM. High hepatitis C incidence in new injecting drug users: A policy failure? Aust N Z J Public Health 2007; 31:30-5.

14. Smyth BP, O’Connor JJ, Barry J, Keenan E. Retrospective cohort study examining incidence of HIV and hepatitis $\mathrm{C}$ infection among injecting drug users in Dublin. J Epidemiol Community Health 2003; 57:310-1.

15. Burke KP, Cox AL. Hepatitis C virus evasion of adaptive immune responses: A model for viral persistence. Immunol Res 2010; 47:216-27.

16. Deuffic-Burban S. Expected increase in prevalence of HCV-related cirrhosis and its complications in the United States: No effect of current antiviral treatment coverage? Gastroenterol Clin Biol 2010; 34:577-9.

17. Grebely J, Thomas DL, Dore GJ. HCV reinfection studies and the door to vaccine development. J Hepatol 2009; 51:628-31.

18. Stoové MA, Gifford SM, Dore GJ. The impact of injecting drug use status on hepatitis C-related referral and treatment. Drug Alcohol Depend 2005; 77:81-6.

19. Stern RK, Hagan H, Lelutiu-Weinberger C, et al. The HCV Synthesis Project: Scope, methodology, and preliminary results. BMC Med Res Methodol 2008; 8:62.

20. Aaron S, McMahon JM, Milano D, et al. Intranasal transmission of hepatitis C virus: Virological and clinical evidence. Clin Infect Dis 2008; 47:931-4.

21. Judd A, Parry J, Hickman M, et al. Evaluation of a modified commercial assay in detecting antibody to hepatitis $\mathrm{C}$ virus in oral fluids and dried blood spots. J Med Virol 2003; 71:49-55.

22. Palmateer N, Kimber J, Hickman M, et al. Evidence for the effectiveness of sterile injecting equipment provision in preventing hepatitis $\mathrm{C}$ and human immunodeficiency virus transmission among injecting drug users: A review of reviews. Addiction 2010; 105:844-59.

23. Wright NM, Tompkins CN. A review of the evidence for the effectiveness of primary prevention interventions for hepatitis $\mathrm{C}$ among injecting drug users. Harm Reduct J 2006; 3:27.

24. Jones L, Pickering L, Sumnal H, McVeigh J, Bellis MA. Optimal provision of needle and syringe programmes for injecting drug users: A systematic review. Int J Drug Policy 2010; 21:335-42.

25. Hallinan R, Byrne A, Dore GJ. Harm reduction, hepatitis C and opioid pharmacotherapy: An opportunity for integrated hepatitis C virusspecific harm reduction. Drug Alcohol Rev 2007; 26:437-43.

26. Tohme RA, Holmberg SD. Is sexual contact a major mode of hepatitis C virus transmission? Hepatology 2010; 52:1497-505.

27. Terrault NA. Sexual activity as a risk factor for hepatitis C. Hepatology 2002; 36:S99-105.

28. Zhang J, Yu KF. What's the relative risk? A method of correcting the odds ratio in cohort studies of common outcomes. JAMA 1998; 280:1690-1. 
29. Greenland S, Thomas DC. On the need for the rare disease assumption in case-control studies. Am J Epidemiol 1982; 116:547-53.

30. Roy E, Alary M, Morissette C, et al. SurvUDI Working Group. High hepatitis $\mathrm{C}$ virus prevalence and incidence among Canadian intravenous drug users. Int J STD AIDS 2007; 18:23-7.

31. Greenland S. Quantitative methods in the review of epidemiologic literature. Epidemiol Rev 1987; 9:1-30.

32. Garfein RS, Golub ET, Greenberg AE, et al. DUIT Study Team. A peereducation intervention to reduce injection risk behaviors for HIV, hepatitis $C$ virus infection in young injection drug users. AIDS 2007; 21:1923-32.

33. Stein MD, Herman DS, Anderson BJ. A trial to reduce hepatitis C seroincidence in drug users. J Addict Dis 2009; 28:389-98.

34. Brunton C, Kemp R, Raynel P, Harte D, Baker M. Cumulative incidence of hepatitis $\mathrm{C}$ seroconversion in a cohort of seronegative injecting drug users. N Z Med J 2000; 113:98-101.

35 Lamothe F, Bruneau J, Franco E, Vincelette J, Lachance N. Prevalence, incidence and risk factors for hepatitis $\mathrm{C}$ infection among injection drug users participating in the Saint-Luc cohort. A report prepared under contract (5498) with the Laboratory Centre for Disease Control, Health Canada. Montreal, Canada: McGill University, 1997.

36. Patrick DM, Tyndall MW, Cornelisse PG, et al. Incidence of hepatitis $\mathrm{C}$ virus infection among injection drug users during an outbreak of HIV infection. CMAJ 2001; 165:889-95.

37. Craine N, Hickman M, Parry JV, et al. Incidence of hepatitis C in drug injectors: The role of homelessness, opiate substitution treatment, equipment sharing, and community size. Epidemiol Infect 2009; 137:1255-65.

38. Crofts N, Nigro L, Oman K, Stevenson E, Sherman J. Methadone maintenance and hepatitis $\mathrm{C}$ virus infection among injecting drug users. Addiction 1997; 92:999-1005.

39. Dolan KA, Shearer J, White B, et al. Four-year follow-up of imprisoned male heroin users and methadone treatment: Mortality, re-incarceration and hepatitis C infection. Addiction 2005; 100:820-8.

40. Hallinan R, Byrne A, Amin J, Dore GJ. Hepatitis C virus incidence among injecting drug users on opioid replacement therapy. Aust N Z J Public Health 2004; 28:576-8.

41. Rezza G, Sagliocca L, Zaccarelli M, et al. Incidence rate and risk factors for HCV seroconversion among injecting drug users in an area with low HIV seroprevalence. Scand J Infect Dis 1996; 28:27-9.

42. Thiede H, Hagan H, Murrill CS. Methadone treatment and HIV and hepatitis $\mathrm{B}$ and $\mathrm{C}$ risk reduction among injectors in the Seattle area. J Urban Health 2000; 77:331-45.

43. van Beek I, Dwyer R, Dore GJ, Luo K, Kaldor JM. Infection with HIV, hepatitis $\mathrm{C}$ virus among injecting drug users in a prevention setting: Retrospective cohort study. BMJ 1998; 317:433-7.

44. Hagan H, Jarlais DC, Friedman SR, Purchase D, Alter MJ. Reduced risk of hepatitis $\mathrm{B}$ and hepatitis $\mathrm{C}$ among injecting drug users participating in the Tacoma syringe-exchange program. Am J Public Health 1995; 85:1531-7.
45. Hagan H, Thiede H, Des Jarlais DC. Hepatitis C virus infection among injection drug users: Survival analysis of time to seroconversion. Epidemiology 2004; 15:543-9.

46. Holtzman D, Barry V, Ouellet LJ, et al. The influence of needle exchange programs on injection risk behaviors and infection with hepatitis $\mathrm{C}$ virus among young injection drug users in select cities in the United States, 1994-2004. Prev Med 2009; 49:68-73.

47. Thorpe LE, Ouellet LJ, Hershow R, et al. Risk of hepatitis C virus infection among young adult injection drug users who share injection equipment. Am J Epidemiol 2002; 155:645-53.

48. Hagan $\mathrm{H}$, Thiede H. Does bleach disinfection of syringes help prevent hepatitis C virus transmission? Epidemiology 2003; 14:628-9; author reply 629.

49. Hagan H, Pouget ER, Garfein RS. Response to Gyarmathy. J Infect Dis 2010; 202:500-1.

50. Hahn JA, Page-Shafer K, Lum PJ, et al. Hepatitis C virus seroconversion among young injection drug users: Relationships and risks. J Infect Dis 2002; 186:1558-64.

51. Kapadia F, Vlahov D, Des Jarlais DC, et al. Second Collaborative Injection Drug User Study (CIDUS-II) Group. Does bleach disinfection of syringes protect against hepatitis $\mathrm{C}$ infection among young adult injection drug users? Epidemiology 2002; 13:738-41.

52. Abou-Saleh M, Davis P, Rice P, et al. The effectiveness of behavioural interventions in the primary prevention of hepatitis $\mathrm{C}$ amongst injecting drug users: A randomised controlled trial and lessons learned. Harm Reduct J 2008; 5:25.

53. Higgins JP, Thompson SG. Quantifying heterogeneity in a metaanalysis. Stat Med 2002; 21:1539-58.

54. Borenstein M. Comprehensive meta-analysis [computer software]. Englewood, NJ: Biostat; 2005.

55. DerSimonian R, Laird N. Meta-analysis in clinical trials. Controlled Clin Trials 1986; 7:177-88.

56. Sterne JA, Egger M. Funnel plots for detecting bias in meta-analysis: Guidelines on choice of axis. J Clin Epidemiol 2001; 54:1046-55.

57. Rhodes T, Kimber J, Small W, et al. Public injecting and the need for 'safer environment interventions' in the reduction of drug-related harm. Addiction 2006; 101:1384-93.

58. Mateu-Gelabert P, Treloar C, Calatayud VA, et al. How can hepatitis C be prevented in the long term? Int J Drug Policy 2007; 18:338-40.

59. Hosmer DL, Lemeshow S. Applied logistic regression. New York: John Wiley \& Sons, 1989.

60. Dales LG, Ury HK. An improper use of statistical significance testing in studying covariables. Int J Epidemiol 1978; 7:373-5.

61. Hagan H, McGough JP, Thiede H, et al. Volunteer bias in nonrandomized evaluations of the efficacy of needle-exchange programs. J Urban Health 2000; 77:103-12.

62. Schechter MT, Strathdee SA, Cornelisse PG, et al. Do needle exchange programmes increase the spread of HIV among injection drug users? An investigation of the Vancouver outbreak. AIDS 1999; 13:F45-51. 